\title{
Concentration profiles of particles settling in the neutral and stratified atmospheric boundary layer
}

\author{
Marcelo Chamecki · René van Hout • \\ Charles Meneveau - Marc B. Parlange
}

Received: 22 December 2006 / Accepted: 24 March 2007 / Published online: 11 May 2007

(C) Springer Science+Business Media B.V. 2007

\begin{abstract}
An expression for the vertical equilibrium concentration profile of heavy particles, including the effects of canopy on the eddy diffusivity as well as corrections for atmospheric stability, is proposed. This expression is validated against measurements of vertical concentration profiles of corn pollen above a corn field. The fitted theoretical profiles show that particle settling is correctly accounted for. The sensitivity to variations in the turbulent Schmidt number, settling velocity and stability corrections are explicitly characterized. The importance of independent measurements of the surface flux of pollen in future experiments is noted.
\end{abstract}

Keywords Atmospheric stability - Corn pollen - Equilibrium profiles . Pollen concentration $\cdot$ Settling velocity $\cdot$ Suspended heavy particles

\footnotetext{
M. Chamecki

Department of Geography and Environmental Engineering, Center for Environmental and Applied Fluid Mechanics, Johns Hopkins University, Baltimore MD, USA

e-mail: chamecki@jhu.edu

R. van Hout

Faculty of Mechanical Engineering, Technion - IIT, Haifa, Israel

e-mail: rene@tx.technion.ac.il

C. Meneveau $(\varangle)$

Department of Mechanical Engineering, Center for Environmental and Applied Fluid Mechanics, Johns Hopkins University, Baltimore MD, USA

e-mail: meneveau@jhu.edu

\section{B. Parlange}

School of Architecture, Civil, and Environmental Engineering, Ecole Polytechnique

Fédérale de Lausanne, Lausanne, Switzerland

e-mail: marc.parlange@epfl.ch
} 


\section{Introduction}

Wind dispersion of airborne pollen (anemophilous) has been a subject of interest for botanists and allergists for a long time (e.g., Aylor and Parlange 1975). More recently, the development of genetically modified crops and questions about cross-pollination and subsequent contamination of natural plant populations has brought even more interest to this field (e.g. Aylor et al. 2003). A crucial question is how far from the source field the pollen grains will be transported and how this distance is affected by turbulence and aerodynamic characteristics of the pollen grain. In order to answer this question, an understanding of the relative importance of gravitational settling, turbulent diffusion and mean wind advection is needed. In this work, we study the effect of gravitational settling, turbulent diffusion and atmospheric stability on the resulting equilibrium concentration profiles of airborne pollen.

Interest in equilibrium (steady state) profiles of suspended particles has a long history and the first analytical results are due to Prandtl (1952). Concerned mostly with profiles of blown snow and sand, as well as sediment transport in rivers, Prandtl assumed a balance between vertical turbulent diffusion and gravitational settling to write

$$
K_{\mathrm{c}} \frac{\mathrm{d} C}{\mathrm{~d} z}+w_{\mathrm{s}} C=0,
$$

where $z$ is the distance from the ground, $C$ is the particle concentration, $K_{\mathrm{c}}$ is the particle eddy diffusivity and $w_{\mathrm{s}}$ is the settling velocity. Modelling the diffusivity as $K_{\mathrm{c}}=\kappa z u_{*}$, where $u_{*}$ is the friction velocity and $\kappa$ is von Karman's constant, integration of (1) yields Prandtl's power law profile

$$
\frac{C}{C_{\mathrm{r}}}=\left(\frac{z}{z_{\mathrm{r}}}\right)^{-w_{s} / \kappa u_{*}},
$$

where $C_{\mathrm{r}}$ is the particle concentration at the reference level $z_{\mathrm{r}}$.

A more rigorous analysis starting from the conservation equation for $C$ and assuming horizontal homogeneity and steady state leads to

$$
\frac{\mathrm{d}}{\mathrm{d} z}\left(K_{\mathrm{c}} \frac{\mathrm{d} C}{\mathrm{~d} z}\right)+w_{\mathrm{s}} \frac{\mathrm{d} C}{\mathrm{~d} z}=0 .
$$

If $w_{\mathrm{s}}$ is assumed to be constant, integration of (3) gives

$$
K_{\mathrm{c}} \frac{\mathrm{d} C}{\mathrm{~d} z}+w_{\mathrm{s}} C=-\Phi,
$$

which is equivalent to (1) if the constant of integration $\Phi$ is assumed to be zero. However, Kind (1992) argued that this assumption cannot be physically justified since $\Phi$ is the net vertical flux resulting from the imbalance between turbulent diffusion and gravitational settling, which are not closely coupled. Kind proposed a more general solution taking into account the non-zero flux

$$
\frac{C}{C_{\mathrm{r}}}=\left(\frac{C_{*}}{C_{\mathrm{r}}} \frac{u_{*}}{w_{\mathrm{s}}}+1\right)\left(\frac{z}{z_{\mathrm{r}}}\right)^{-w_{\mathrm{s}} / \kappa u_{*}}-\frac{C_{*}}{C_{\mathrm{r}}}\left(\frac{u_{*}}{w_{\mathrm{s}}}\right) .
$$

where $C_{*}=\Phi / u_{*}$. Expression (5) reduces to the power law when $\Phi=0$.

Kind (1992) presented some experimental evidence based on previous data for blown snow published by other authors suggesting that the data trends were better predicted by Eq. (5) than Eq. (2). However, his analysis was based on crude estimates for the ratio $C_{\mathrm{r}} / C_{*}$. The 
strongest point in favour of Kind's theory is that it recovers the well-known logarithmic profile (e.g., Monin 1970) in the limit $w_{s} \rightarrow 0$ :

$$
\frac{C}{C_{\mathrm{r}}}=1-\frac{C_{*}}{\kappa C_{\mathrm{r}}} \ln \left(\frac{z}{z_{\mathrm{r}}}\right) .
$$

Not many authors have tried to verify the applicability of Kind's expression, most probably because of difficulties in measuring the surface flux of particles. Gillies and Berkofsky (2004) used measured profiles of suspended dust to assess the validity of expressions (2), (5) and (6). Their comparisons show that the logarithmic profile fits the data better than the power law. Although the authors do not explicitly mention it, one can conclude from their work that for small dust particles, neglecting gravitational settling is less problematic than neglecting the non-zero surface flux. In Gillies and Berkofsky (2004) no explicit evaluation of the profile predicted by Kind (which includes both effects) is made. Their analysis is restricted to verifying the range of variation of the parameter $C_{\mathrm{r}} / C_{*}$.

Xiao and Taylor (2002) performed single column numerical simulations of blown snow profiles. Their results show that as the steady state condition is approached the surface flux stabilizes at values $\Phi \neq 0$, confirming the importance of retaining this term. Furthermore, they also noted that the approximation $\Phi=0$ is better for larger particles. Their analysis of vertical concentration profiles indicates that Eq. (5) is better than the logarithmic profile (6), but their results display some dependence on the imposed upper boundary conditions.

In this work, we modify Kind's profile (5) to account for changes in the eddy diffusivity induced by the canopy as well as variations in the atmospheric stability. The applicability of the resulting equations to vertical concentration profiles of suspended corn pollen grains measured in a field experiment is investigated.

\section{Eddy diffusivity and atmospheric stability}

Before expression (5) can be applied to predict the concentration of airborne pollen grains, some modifications are needed. First, Kind (1992) assumed that the eddy diffusivity for particles is the same as for momentum and that the latter is given by $K_{\mathrm{m}}=\kappa z u_{*}$. Since in this case we are interested in the flow over canopies, the momentum diffusivity is modified by the introduction of the displacement height $(d)$ and becomes $K_{\mathrm{m}}=\kappa(z-d) u_{*}$ (e.g., Brutsaert 1982; Kaimal and Finnigan 1994). On the other hand, all the evidence points to the fact that the eddy diffusivity for scalars is larger than for momentum in the canopy roughness layer $d \leq z \lesssim 3 h$, where $h$ is the canopy height (e.g., Finnigan 2000). The difference in diffusivities can be accounted for by introducing a Schmidt number $(S c)$ thus expressing the eddy diffusivity as

$$
K_{\mathrm{c}}=\frac{\kappa}{S c}(z-d) u_{*},
$$

where $S c$ can be a function of $z$.

In deriving Eq. (5), Kind also assumed neutral atmospheric stratification arguing that when aeolian transport occurs wind speeds are relatively high and neutral stratification is a good approximation. Although neutral stability is a good starting point, significant transport occurs under stable stability and especially during daytime under unstable conditions. Thus the influence of stability on the shape of the profiles has to be taken into consideration. Traditionally the effects of atmospheric stability on surface-layer phenomena are represented by using Monin-Obukhov similarity theory (Monin and Obukhov 1954). For passive scalars, 
the way to proceed is to express the vertical dimensionless gradient of the concentration as a function of the distance to the ground and the Obukhov length $L$,

$$
\frac{1}{\Phi}\left(K_{\mathrm{c}} \frac{\mathrm{d} C}{\mathrm{~d} z}\right)=\frac{\kappa(z-d)}{S c C_{*}} \frac{\mathrm{d} C}{\mathrm{~d} z}=-\phi_{\mathrm{c}}\left(\frac{z-d}{L}\right),
$$

where $\phi_{c}$ is the similarity function for concentration. Note that the left-hand side of the expression emphasizes the fact that the similarity function is equivalent to the turbulent flux normalized by the surface flux.

By analogy we may write the dimensionless vertical flux for particle concentration (now composed of turbulent and settling parts) as

$$
\frac{1}{\Phi}\left(K_{\mathrm{c}} \frac{\mathrm{d} C}{\mathrm{~d} z}+w_{\mathrm{s}} C\right)=\frac{\kappa(z-d)}{S c C_{*}} \frac{\mathrm{d} C}{\mathrm{~d} z}+\frac{w_{\mathrm{s}}}{u_{*}} \frac{C}{C_{*}}=-\phi_{c}\left(\frac{z-d}{L}\right) .
$$

Integration of expression (9) requires the specification of functions for $S c$ and $\phi_{c}$. At this point this would have to be done based on experimental data and requires measurements of the surface fluxes. In order to be able to proceed in the absence of such data, we first assume that the diffusivity and the Schmidt number for pollen dispersion are the same as for other scalars such as temperature and humidity. Finnigan (2000) suggested that the diffusivity for scalars is about two or three times larger than $K_{\mathrm{m}}=\kappa(z-d) u_{*}$ at canopy height and approximately equal to $K_{\mathrm{m}}$ at the top of the roughness layer $(z \approx 3 h)$. As a first approximation, we assume a constant value for the Schmidt number within the entire roughness layer. Li and Taylor (2005) suggest that, based on their three-dimensional inertial Lagrangian stochastic model, a constant Schmidt number used together with the settling velocity in still fluid may be a good approximation. We further assume that $\phi_{c}((z-d) / L)=\phi_{c}(\zeta)$ can be represented by the traditional Businger-Dyer relations for passive scalars (Businger et al. 1971; Dyer 1974):

$$
\phi_{c}(\zeta)= \begin{cases}(1-16 \zeta)^{-1 / 2} & \zeta<0 \\ 1+5 \zeta & \zeta>0\end{cases}
$$

There are many questions whether the Businger-Dyer functions (or other similar expressions) are valid for momentum and heat fluxes above canopies (e.g. Raupach 1979; Finnigan 2000). We reproduce in Fig. 1 the data from Raupach (1979) and Viswanadham et al. (1987) above temperate and tropical forests as assembled by Shuttleworth (1989). Fig. 1 a shows good agreement between the data and the similarity functions for momentum $\left(\phi_{m}\right)$. In Fig. 1b the data and similarity functions for both temperature $\left(\phi_{h}\right)$ and humidity $\left(\phi_{q}\right)$ are shown. As noted by Raupach (1979), the similarity functions (10) for scalars overpredict the dimensionless fluxes by a factor of two. Note that for passive scalars, adopting a similarity function equal to $(1 / 2) \phi_{c}$ is the same as using $S c=1 / 2$ and keeping the traditional form of $\phi_{c}$. We adopt the latter and assume $S c=1 / 2$ within the roughness layer.

Using the similarity functions (10) and assuming $S c$ to be constant, Eq. (9) can be integrated (details are presented in the Appendix A) to obtain

$$
\frac{C}{C_{\mathrm{r}}}=\left[\frac{C_{*}}{C_{\mathrm{r}}} \frac{u_{*}}{w_{\mathrm{s}}} \Omega\left(\frac{z_{\mathrm{r}}-d}{L}\right)+1\right]\left(\frac{z-d}{z_{\mathrm{r}}-d}\right)^{-\alpha}-\left(\frac{C_{*}}{C_{\mathrm{r}}}\right) \frac{u_{*}}{w_{\mathrm{s}}} \Omega\left(\frac{z-d}{L}\right)
$$

where $\alpha=w_{\mathrm{s}} S c / \kappa u_{*}$ is a measure of the ratio settling/diffusivity. The stability corrections $\Omega(\zeta)$ are given by

$$
\Omega(\zeta)= \begin{cases}{ }_{2} F_{1}(\alpha, 1 / 2 ; 1+\alpha ; 16 \zeta) & \zeta<0 \\ 1+5\left(\frac{\alpha}{\alpha+1}\right) \zeta & \zeta>0\end{cases}
$$



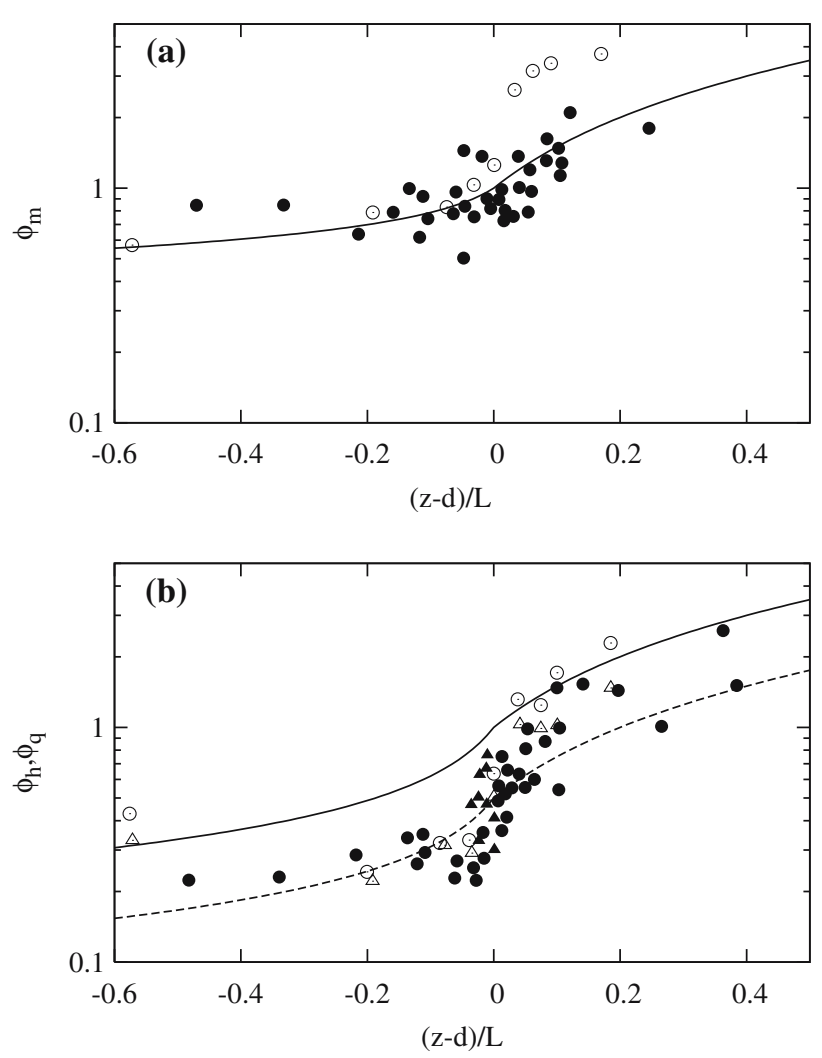

Fig. 1 Similarity functions of (a) velocity and (b) temperature (circles) and humidity (triangles). Full symbols from temperate forest and open ones from tropical forest (adapted from Shuttleworth (1989)). Solid lines are the Businger-Dyer functions and dashed line is $(1 / 2) \phi_{c}$, which can be interpreted as $S c=1 / 2$

Here ${ }_{2} F_{1}(a, b ; c ; x)$ is the Gauss hypergeometric function. In practice, the approximation for the hypergeometric function proposed by Butler and Wood (2002) and presented in the Appendix B can be used. Equation (11) can be considered a generalization of Kind's expression including the effects of the canopy and atmospheric stability.

It is worth mentioning that if expressions (12) are substituted into equation (11) and the limit $w_{s} \rightarrow 0$ is taken, the traditional Monin-Obukhov expressions for passive scalars are recovered.

For neutral conditions Eq. (11) reduces to

$$
\frac{C}{C_{\mathrm{r}}}=\left(\frac{C_{*}}{C_{\mathrm{r}}} \frac{u_{*}}{w_{\mathrm{s}}}+1\right)\left(\frac{z-d}{z_{\mathrm{r}}-d}\right)^{-\alpha}-\left(\frac{C_{*}}{C_{\mathrm{r}}}\right) \frac{u_{*}}{w_{\mathrm{s}}} .
$$

This is Kind's equation with the eddy diffusivity given by (7), which will be further studied in the next section. 


\section{Experimental results}

A field experiment to measure airborne corn pollen concentration and turbulence statistics took place in July 2004 close to Queenstown, Maryland. The cornfield was located in a flat region and was approximately $900 \times 300 \mathrm{~m}^{2}$. The average height of the corn was $h=3 \mathrm{~m}$. Pollen concentrations were measured approximately at the centre of the field at four different heights $(z=3,3.75,4.5$ and $6 \mathrm{~m}$ ) using Rotorod (Multidata) rotating impact samplers (each sampler provided two independent measurements). Momentum and heat fluxes were measured with 3D sonic anemometers (Campbell Scientific) at two heights ( $z=3 \mathrm{~m}$ and $4.5 \mathrm{~m}$ ) along with standard meteorological measurements. The experiment is explained in detail by R. van Hout et al. (Priv. Comm. 2006).

Here only data from the days when pollen concentrations were high and eddy correlation measurements were available are used (July 16th, 17th and 19th). Sampling intervals were $1 \mathrm{~h}$ in the morning (0600-1200 Eastern Daylight saving Time (EDT)) and $2 \mathrm{~h}$ in the afternoon (1200-1800 EDT). Pollen concentrations always peaked during the morning hours, decaying during the afternoon. Some early morning or late afternoon sampling periods had very small concentrations and those measurements were considered statistically unreliable (i.e. the difference in pollen counts between the two independent rods of each sampler was large). To avoid problems, only sampling periods displaying concentration $C(z=h)>10$ grains $\mathrm{m}^{-3}$ are used in this work and concentration values are the average of the two rods during the same sampling period. As an example, the diurnal evolution of pollen concentrations for July 16th is shown in Fig. 2a. For a detailed analysis of the experimental results, the reader is referred to R. van Hout et al. (Priv. Comm. 2006).

As a first step, neutral conditions are assumed and the applicability of Eq. (13) is tested. The displacement height is assumed to be $d=(2 / 3) h$ as suggested by Brutsaert (1982), and $S c=1 / 2$ as mentioned in the previous section. The settling velocity is assumed to be the same as the terminal velocity in still air and is estimated using Stokes law while assuming a spherical pollen grain. Here the value for corn pollen grains $w_{\mathrm{s}}^{t}=0.27 \mathrm{~m} \mathrm{~s}^{-1}$ estimated by R. van Hout et al. (Priv. Comm. 2006) is used. This value was obtained using the corn pollen density and diameter measured by van Hout and Katz (2004). Note that the pollen grain diameter used $\left(d_{\mathrm{p}}=89.2 \mu \mathrm{m}\right.$ with $\left.\sigma_{d_{\mathrm{P}}}=3.8 \mu \mathrm{m}\right)$ is for fully hydrated grains (i.e. immersed in water). Under field conditions the water content of the pollen grains may decrease depending on the elapsed time after release from the anther. Consequently, the pollen diameter may decrease leading to somewhat smaller settling velocities (Aylor 2002). Pollen size distributions from the field where the experiment was conducted are not very different and are discussed by R. van Hout et al. (Priv. Comm. 2006).

Since independent measurements of the surface pollen flux $\left(\Phi=C_{*} u_{*}\right)$ are not available, to each profile the flux and the reference concentration $\left(C_{\mathrm{r}}\right)$ at $z_{\mathrm{r}}=3 \mathrm{~m}$ are fit in Eq. (13) by minimizing the mean square error (MSE) between the profile and the data. The Matlab implementation of the simplex search method of Lagarias et al. (1998) is used. Note that although values of $C_{\mathrm{r}}$ are available from the field data, they are not imposed (i.e. they are also fitted). The MSE is then determined based on the four measurement heights available and the fitted profile is not required to pass through any of the data points. Tests performed by actually imposing $C_{\mathrm{r}}$ and calculating the MSE based on the three remaining points yield very similar results, with slightly larger MSE values.

The experimental data (together with error bar estimates based on the two concentration measurements available at each height) and the fitted profiles for July 16th are shown in Fig. 3. The corresponding time series obtained for the surface flux is presented in Fig. $2 b$. Positive flux values are associated with upward fluxes and negative values (such as the one 

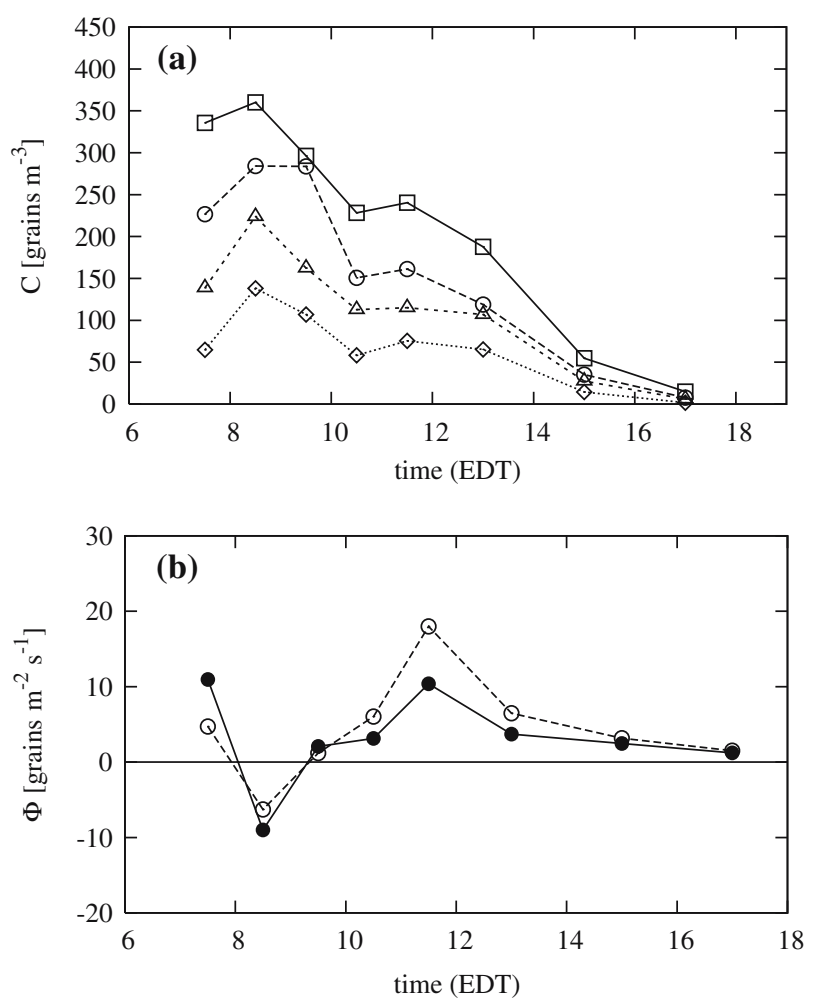

Fig. 2 (a) measured pollen concentrations for July 16th at: $z=3 \mathrm{~m}(\square), z=3.75 \mathrm{~m}(\circ), z=4.5 \mathrm{~m}(\triangle)$ and $z=6 \mathrm{~m}(\diamond)$ and $(\mathbf{b})$ estimated surface pollen fluxes using the neutral profile $(\bullet)$ and including stability correction (०)

obtained for the second run of July 16th) correspond to surface deposition. It is important to note that, in the field experiment being analyzed where the only pollen source is the underlying homogeneous field, negative fluxes do not make sense under equilibrium conditions. The fact that a negative value was obtained will be discussed later.

The main difference between concentration profiles of pollen grains (or other heavy particles) and scalars such as temperature and humidity is the effect of gravitational settling. It is therefore of interest to investigate whether this effect is correctly incorporated in expression (13). To approach this question without independent measurements of the surface fluxes, profiles are fitted to the data (in the same way as before) using different values of settling velocity. Each value of settling velocity yields optimal values of $C_{\mathrm{r}}$ and $\Phi$, as well as an optimal mean square error. The output of this procedure is a relationship between the settling velocity used and the corresponding optimal MSE obtained for each sampling period. Results for July 16th, 17th and 19th are shown in Fig. 4, where the normalized optimal mean square error $\left(\mathrm{MSE} / C_{\mathrm{r}}^{2}\right)$ is plotted as a function of the ratio between settling velocity $\left(w_{\mathrm{s}}\right)$ and terminal velocity in still air $\left(w_{\mathrm{s}}^{\mathrm{t}}\right)$.

If the effect of gravitational settling is correctly represented by the theoretical profile, the MSE would be minimum for the correct value of the settling velocity (in the absence of experimental errors). As can be noted, most of the curves are marked by a significant decrease in the mean square error near $w_{\mathrm{s}} / w_{\mathrm{s}}^{\mathrm{t}}=1$. In fact, from the 19 runs available, 12 

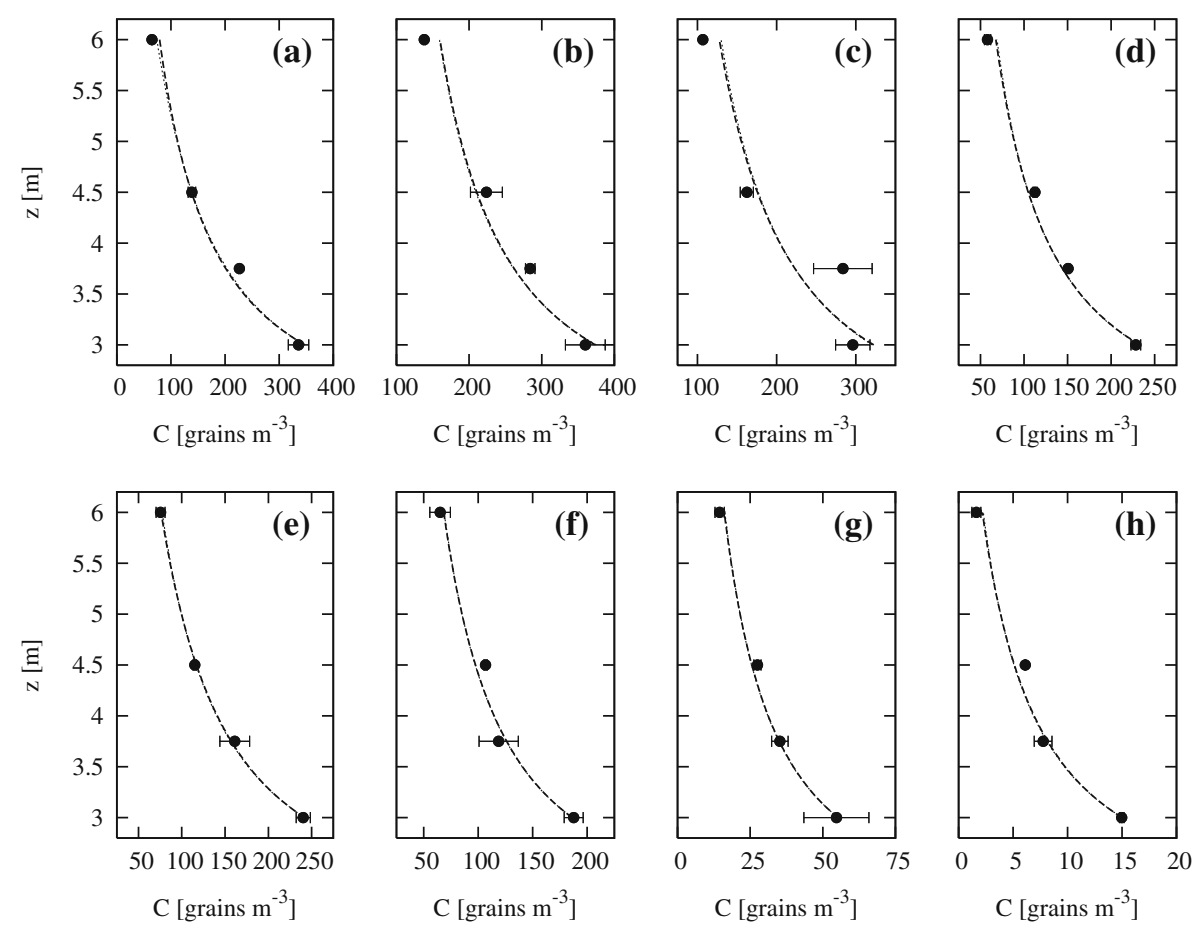

Fig. 3 Vertical concentration profiles: measured data (•), neutral fits (dashed lines) and stability-corrected fits (dotted lines-almost indistinguishable) for July 16th (a) 0700-0800 EDT (b) 0800-0900 EDT (c) 0900-1000 EDT (d) 1000-1100 EDT (e) 1100-1200 EDT (f) 1200-1400 EDT (g) 1400-1600 EDT and (h) 1600-1800 EDT

have the optimal fit (indicated by the dots) within the range $1 / 2<w_{\mathrm{s}} / w_{\mathrm{s}}^{\mathrm{t}}<2$. This is good agreement, especially if one realizes that the settling velocity used as reference is estimated for still air and does not take in account the effect of turbulence (e.g., Wang and Maxey 1993). Pollen size distribution and uncertainties in hydration level of the pollen grains and the value of the Schmidt number contribute to the scatter. Note that only in three cases the smallest value of $w_{\mathrm{s}}$ yields the best result, meaning that only in these three cases the logarithmic profile assuming $w_{\mathrm{s}}=0$, describes the data better than Eq. (13). Efforts to correlate these cases with atmospheric stability, friction velocity, or other important characteristic parameters did not yield any conclusive results. The results presented in Fig. 4 provide substantial evidence that the theoretical profile represented by Eq. (13) captures the effects of gravitational settling correctly. The fact that the surface fluxes (Fig. 2b) are different from zero also confirms the importance of retaining this term in the Eq. (4).

At this point it is important to recognize that the uncertainty in the value of the Schmidt number, the settling velocity or the form of the stability correction may have an important effect on the results presented so far. A natural question that therefore arises is how sensitive the results are to changes in these parameters. With respect to the Schmidt number, although the value $S c=1 / 2$ seems to be an obvious choice based on Fig. 1, several values have been suggested based on different datasets. In Fig. 5a we plot the optimal values of the ratio $w_{\mathrm{s}} / w_{\mathrm{s}}^{t}$ for each run using $S c=1,1 / 2$ and $1 / 3$ (the change for the same run is highlighted by connected dotted lines). It is clear that increasing the turbulent diffusion (i.e. decreasing 

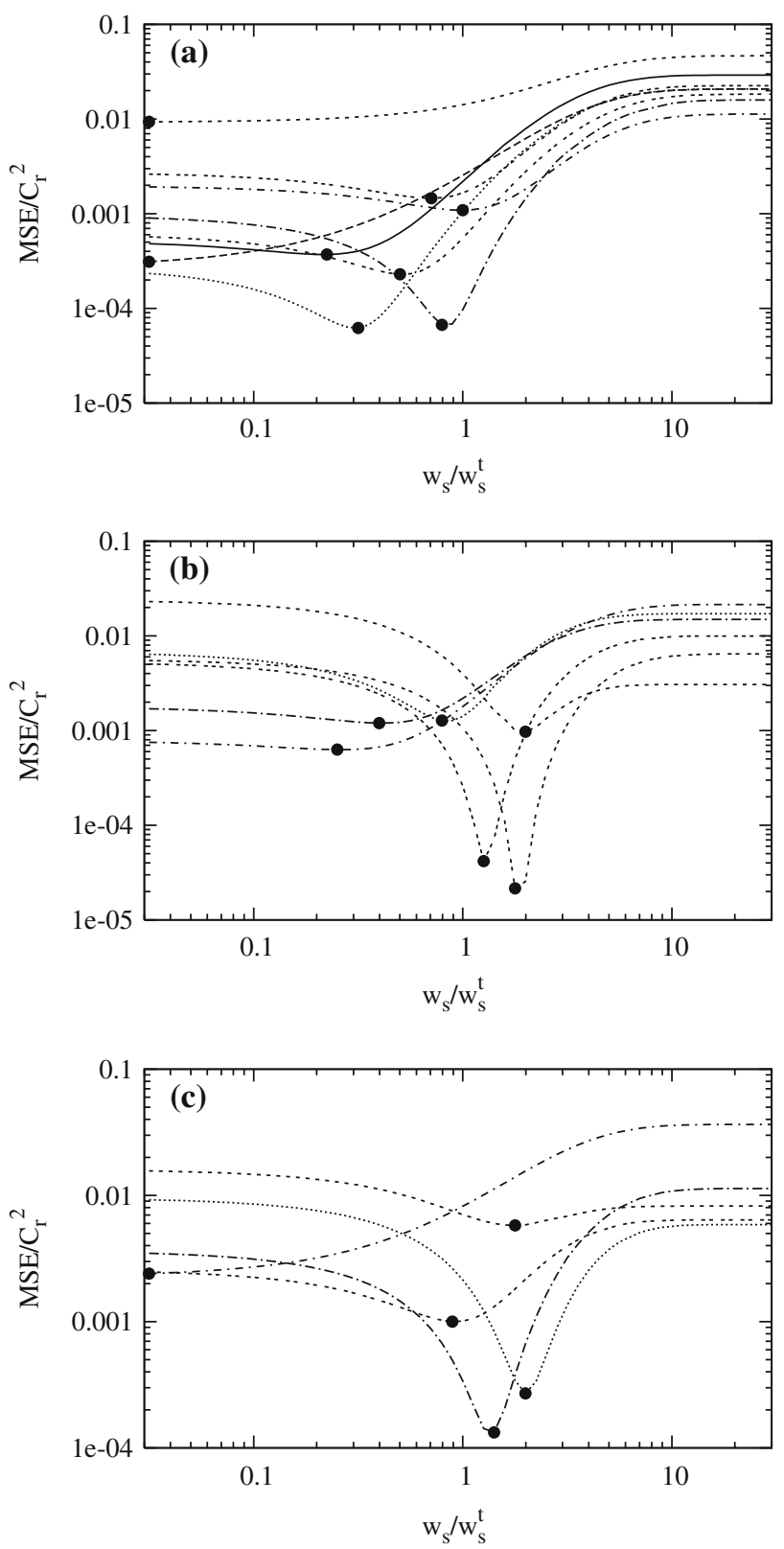

Fig. 4 Normalized mean square error of the fitted profiles as a function of the settling velocity ratio for each run of (a) July 16th, (b) July 17th, and (c) July 19th. The solid circles mark the minimum MSE

the Schmidt number) also increases the optimal value of the settling velocity. Although fits using the terminal velocity $w_{\mathrm{s}}^{t}$ become worse for $S c=1$ or $S c=1 / 3$ (i.e. the MSE becomes larger), if the optimal settling velocity is used the quality of the fits is independent of the Schmidt number and it is difficult from these data to decide upon an adequate value of $S c$. In Fig. 5b the time series of surface pollen fluxes for each value of $S c$ and $w_{\mathrm{s}}^{t}=0.27 \mathrm{~m} \mathrm{~s}^{-1}$ 

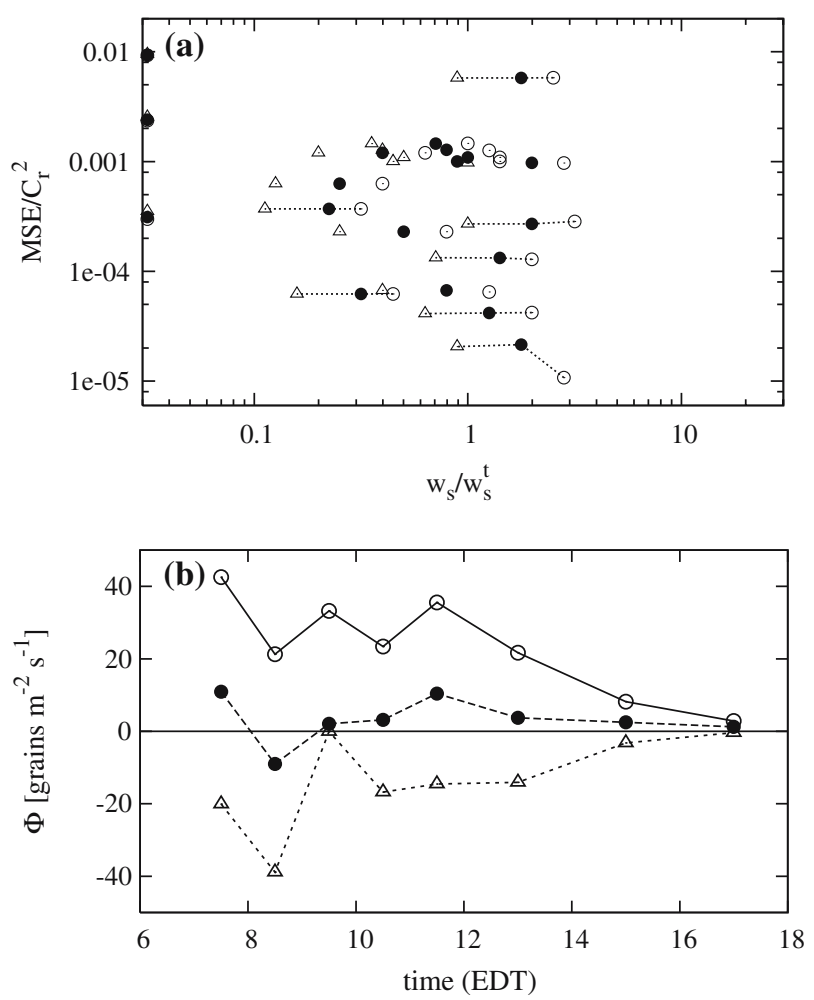

Fig. 5 Effect of the Schmidt number with $S c=1(\triangle), S c=1 / 2(\bullet)$, and $S c=1 / 3(\circ)$. (a) Optimal value of settling velocity ratios for all 19 runs and (b) fitted surface fluxes for July 16th. Note: dotted lines in panel (a) connect points for the same run

are shown. It is clear that the value of the fluxes obtained is very sensitive to changes in $S c$, and this could be used to determine the value of $S c$ when measurements of $\Phi$ are available (and $w_{\mathrm{s}}$ is known). In a similar way, one can analyze the sensitivity of the fitted surface flux to the prescribed settling velocity. In Fig. 6, the obtained surface flux time series are plotted for the terminal velocity $w_{\mathrm{s}}^{t}$ and for values $25 \%$ larger and smaller. Variations of the settling velocity due to effects of turbulence and size distribution of pollen grains can be even larger than that.

The next step is to evaluate the effect of the stability corrections on the shape of the profiles and on the quality of the fits. Equation (11) can be fitted to the data in the same way as Eq. (13), but now taking into account the measured value for the Obukhov length. The resulting profiles for July 16th are presented in Fig. 3 and almost no difference is observed between them and the neutral fits (the average mean square error is slightly smaller, but the difference is too small to draw any conclusions). However, one should note that although the resulting profiles are almost similar, the obtained surface fluxes are quite different. This fact is evident in the time series of the fluxes, shown in Fig. 2b. Although based on the present data we cannot adequately judge if the stability correction (mostly the Businger-Dyer functions) is correct, the large differences found between fluxes (up to 100\%) suggest that this may be an important issue that should be explored in future studies.

To further stress the importance of the stability corrections, we investigate differences between profiles when the surface parameters $\left(C_{\mathrm{r}}\right.$ and $\left.\Phi\right)$ are kept constant. As an example, 


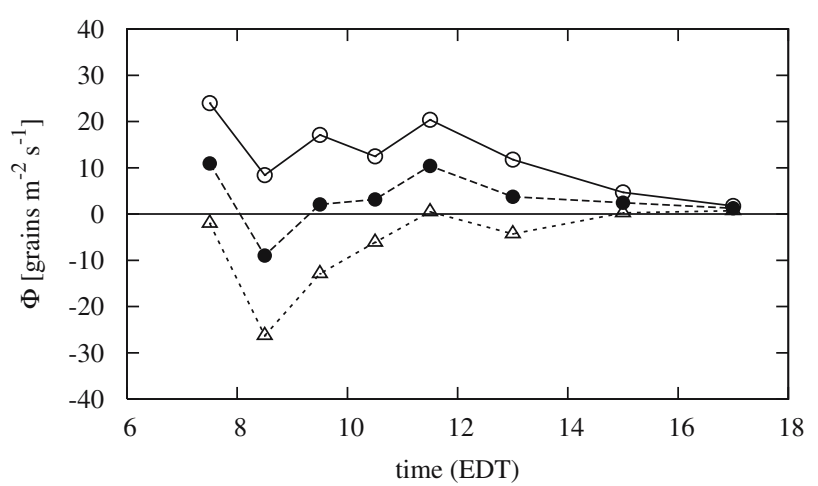

Fig. 6 Effect of the settling velocity on the fitted surface fluxes for July 16th. $w_{\mathrm{s}}=0.75 w_{\mathrm{S}}^{t}(\circ), w_{\mathrm{s}}=w_{\mathrm{S}}^{t}(\bullet)$, and $w_{\mathrm{s}}=1.25 w_{\mathrm{S}}^{t}(\triangle)$

we use the data from the period between 1100 and 1200 EDT for July 16 th: $u_{*}=0.52 \mathrm{~m} \mathrm{~s}^{-1}$, $C_{\mathrm{r}}=240$ grains $\mathrm{m}^{-3}$ and $\Phi=11$ grains $\mathrm{m}^{-2} \mathrm{~s}^{-1}$ (arbitrarily taken from the neutral fit). Profiles are calculated for neutral, unstable $(L=-30 \mathrm{~m})$ and stable $(L=30 \mathrm{~m})$ atmospheric conditions. Both the logarithmic prediction (6) and Eq. (11) are shown in Fig. 7. It is clear that for the present data (i.e. heavy pollen grains) the effect of gravitational settling on the profiles is very strong. However, more important here, Fig. 7 suggests that the effect of the stability corrections in the case of heavy particles is important.

\section{Conclusions}

A new expression for equilibrium concentration profiles of particles settling in the atmospheric surface layer has been proposed. This is an extension to the expression proposed by Kind (1992) to incorporate the effect of the canopy on the eddy diffusivity, as well as the influence of atmospheric stability. In the derivation of the proposed expressions, a constant Schmidt number and the applicability of the Businger-Dyer functions for suspended particles are assumed.

Corn pollen concentrations above a cornfield are used to verify the validity of the proposed theoretical profile. The theoretical profiles are fitted to the experimental data and show good agreement. It is observed that the mean square error is minimized near the still air settling velocity providing evidence that the effect of gravitational settling has been correctly incorporated into the proposed expression. The importance of considering the corrections for atmospheric stability is clearly exhibited.

The final expression aims to predict the vertical concentration profile under conditions where settling velocity, Schmidt number, atmospheric stability (and the appropriate shape for the similarity functions), surface particle flux, displacement height and friction velocity are known. Other applications are also possible, in the same way as the log-law has been traditionally used to determine the displacement height and the surface roughness from measured velocity profiles. However, one has to be careful in such applications, since determination of a given parameter can depend critically on the values assigned to the other quantities. Sensitivity tests performed show for example a strong dependence of the fitted surface flux on the adopted Schmidt number, settling velocity as well as on the corrections for atmospheric stability. Additional experiments, including simultaneous measurements of the particle surface 

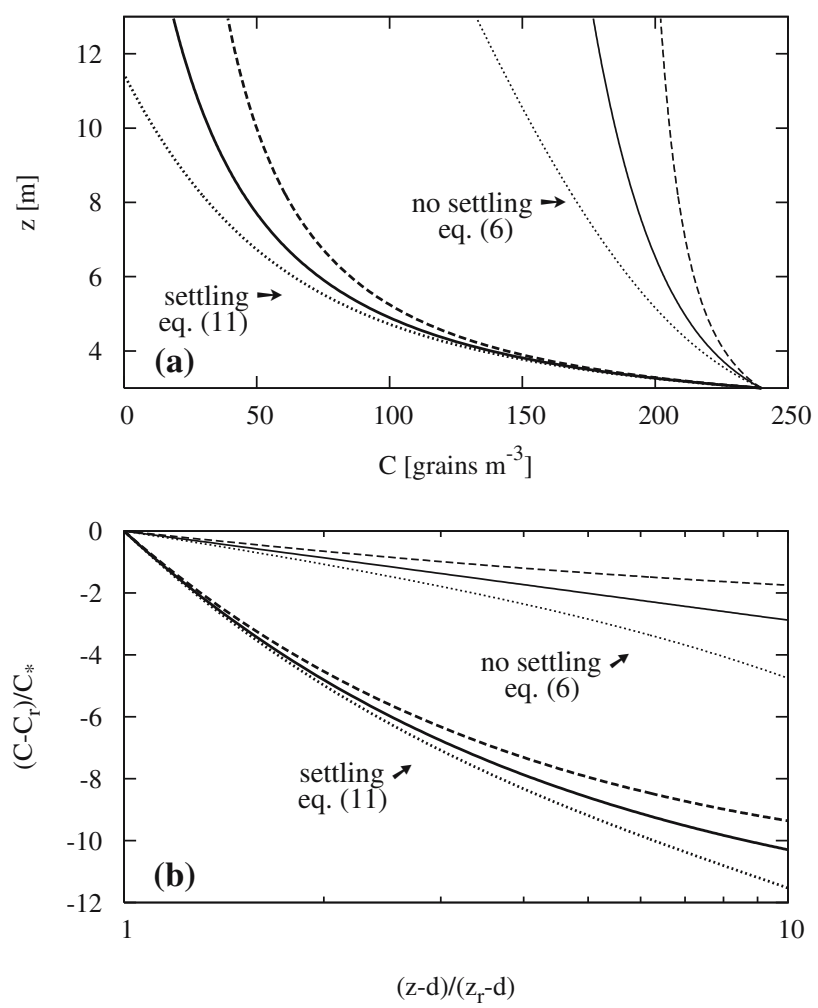

Fig. 7 Influence of stability corrections on the logarithmic profile (6) and on the proposed profile (11). (a) arithmetic scale and (b) traditional logarithmic scale. Neutral profiles (continuous lines), unstable (dotted lines) and stable (dashed lines)

fluxes, atmospheric stability, and all the other relevant quantities are required to determine the appropriate value (or functional dependence) for the Schmidt number and to verify whether the Businger-Dyer functions can be used for heavy particles such as pollen grains.

Acknowledgements This work was supported by the Biocomplexity Program of the National Science Foundation grant 0119903. The authors are grateful to Mike Embry at the Wye Research and Education Center of the University of Maryland for helping to find an appropriate cornfield and to Joe Smith and Chad Higgins for their assistance during the field work.

\section{Appendix A}

Integration of Eq. (9) is done by introducing $\zeta=(z-d) / L$ and rearranging the terms as:

$$
\frac{\mathrm{d} C}{\mathrm{~d} \zeta}+\frac{\alpha}{\zeta} C=-\frac{S c C_{*}}{\kappa \zeta} \phi_{c}(\zeta)
$$

which is a simple form of the Bernoulli differential equation and has the solution

$$
C(L \zeta+d)=\left(\frac{\zeta}{\zeta_{r}}\right)^{-\alpha}\left[-\frac{S c C_{*}}{\kappa} I\left(\zeta ; \zeta_{r}\right)+C_{\mathrm{r}}\right],
$$


where

$$
I\left(\zeta ; \zeta_{r}\right)=\frac{1}{\zeta_{r}} \int_{\zeta_{r}}^{\zeta}\left(\frac{\eta}{\zeta_{r}}\right)^{\alpha-1} \phi_{c}(\eta) \mathrm{d} \eta
$$

$\eta$ is an integration variable and the Schmidt number is assumed to be constant. Equation (16) is the starting point if similarity functions different from the Businger-Dyer equations are to be used.

If $\phi_{c}(\eta)=(1+5 \eta)$ is used for stable conditions, expression (16) is easily integrated to

$$
I\left(\zeta ; \zeta_{r}\right)=\frac{1}{\alpha}\left[\left(\frac{\eta}{\zeta_{r}}\right)^{\alpha}\left(1+\frac{5 \alpha}{\alpha+1} \eta\right)\right]_{\zeta_{r}}^{\zeta} .
$$

For unstable conditions, if $\phi_{c}(\eta)=(1-16 \eta)^{-1 / 2}$ is used one obtains:

$$
I\left(\zeta ; \zeta_{r}\right)=\frac{1}{\alpha}\left[\left(\frac{\eta}{\zeta_{r}}\right)^{\alpha}{ }_{2} F_{1}(\alpha, 1 / 2 ; 1+\alpha ; 16 \eta)\right]_{\zeta_{r}}^{\zeta} .
$$

Substitution of both integrals into Eq. (15) and transforming back from $\zeta$ to $z$ yields expressions (11) and (12).

Also note that Eq. (11) can be written in the following possibly more useful form:

$$
\left[C(z)+\frac{C_{*} u_{*}}{w_{\mathrm{s}}} \Omega\left(\frac{z-d}{L}\right)\right](z-d)^{\alpha}=\mathcal{K},
$$

where $\mathcal{K}$ is a constant completely determined in terms of quantities at the reference level:

$$
\mathcal{K}=\left[C_{\mathrm{r}}+\frac{C_{*} u_{*}}{w_{\mathrm{s}}} \Omega\left(\frac{z_{\mathrm{r}}-d}{L}\right)\right]\left(z_{\mathrm{r}}-d\right)^{\alpha} .
$$

\section{Appendix B}

The stability correction for the profile under unstable atmospheric conditions is given by the Gauss hypergeometric function defined as

$$
{ }_{2} F_{1}(a, b ; c ; x)=\sum_{k=0}^{\infty} \frac{(a)_{k}(b)_{k}}{(c)_{k}} \frac{x^{k}}{k !},
$$

where $(\lambda)_{0}=1$ and $(\lambda)_{k}=\Gamma(\lambda+k) / \Gamma(\lambda)$ and $\Gamma$ is the gamma function (e.g., Lebedev 1972). In practice the function can be evaluated using a numerical procedure (e.g., Press et al. 1997 p. 263) or the Laplace approximation presented by Butler and Wood (2002):

$$
{ }_{2} F_{1}(a, b ; c ; x) \approx c^{c-1 / 2} r_{2,1}^{-1 / 2}\left(\frac{\hat{y}}{a}\right)^{a}\left(\frac{1-\hat{y}}{c-a}\right)^{c-a}(1-x \hat{y})^{-b},
$$

where

$$
\begin{aligned}
\tau & =x(b-a)-c, \\
\hat{y} & =\frac{2 a}{\sqrt{\tau^{2}-4 a x(c-b)}-\tau}, \\
r_{2,1} & =\frac{\hat{y}^{2}}{a}+\frac{(1-\hat{y})^{2}}{(c-a)}-\frac{b x^{2}}{(1-x \hat{y})^{2}} \frac{\hat{y}^{2}}{a} \frac{(1-\hat{y})^{2}}{(c-a)} .
\end{aligned}
$$


Tests were performed with combinations of typical values for $u_{*}, w_{s}, S c$ and $(z-d) / L$ and the error introduced in the profiles $C(z)$ by using Eqs. (22) and (23) instead of numerical approximations is very small.

\section{References}

Aylor DE (2002) Settling speed of corn (Zea mays) pollen. J Aerosol Sci 33:1601-1607

Aylor DE, Parlange JY (1975) Ventilation required to entrain small particles from leaves. Plant Physiol 56:9799

Aylor DE, Schultes NP, Shields EJ (2003) An aerobiological framework for assessing cross-pollination in maize. Agric For Meteorol 119:111-129

Brutsaert W (1982) Evaporation into the atmosphere. D Reidel, Dordrecht, 299 pp

Businger JA, Wyngaard JC, Izumi Y, Bradley EF (1971) Flux-profile relationships in the atmospheric surface layer. J Atmos Sci 28:181-189

Butler RW, Wood ATA (2002) Laplace approximations for hypergeometric functions with matrix argument. Ann Statist 30:1155-1177

Dyer AJ (1974) A review of the flux-profile relationships. Boundary-Layer Meteorol 7:363-372

Finnigan J (2000) Turbulence in plant canopies. Annu Rev Fluid Mech 32:519-571

Gillies JA, Berkofsky L (2004) Eolian suspension above the saltation layer, the concentration profile. J Sediment Res 74:176-183

Kaimal JC, Finnigan JJ (1994) Atmospheric boundary layer flows: their structure and measurement. Oxford University Press, New York 289 pp

Kind RJ (1992) One-dimensional aeolian suspension above beds of loose particles - a new concentrationprofile equation. Atmos Environ A 26:927-931

Lagarias JC, Reeds JA, Wright MH, Wright PE (1998) Convergence properties of the nelder-mead simplex method in low dimensions. SIAM J Optimiz 9:112-147

Lebedev NN (1972) Special functions \& their applications. Dover publications, $308 \mathrm{pp}$

Li PY, Taylor PA (2005) Three-dimensional Lagrangian simulation of suspended particles in the neutrally stratified atmospheric surface layer. Boundary-Layer Meteorol 116:301-311

Monin AS (1970) The atmospheric boundary layer. Annu Rev Fluid Mech 2:225-250

Monin AS, Obukhov AM (1954) Basic laws of turbulent mixing in the ground layer of the atmosphere. Trans Geophys Inst Akad Nauk USSR 151:163-187

Prandtl L (1952) Essentials of fluid dynamics. Blackie and Son, London $452 \mathrm{pp}$

Press WH, Teukolsky SA, Vetterling WT, Flannery BP (1997) Numerical recipes in Fortran 77. Cambridge University Press, 933 pp

Raupach MR (1979) Anomalies in flux-gradient relationships over forest. Boundary-Layer Meteorol 16:467486

Shuttleworth WJ (1989) Micrometeorology of temperate and tropical forest. Phil Trans Roy Soc London B 324:299-334

van Hout R, Katz J (2004) A method for measuring the density of irregularly shaped biological aerosols such as pollen. J Aerosol Sci 35:1369-1384

Viswanadham Y, Sa LDA, Filho VPS, Manzi AO (1987) Evaporation ratios of eddy transfer coefficients over the Amazon forest. In: Swanson RM, Bernier PY, Woodard PD (eds) Forest hydrology and watershed management. IAHS Press

Wang LP, Maxey MR (1993) Settling velocity and concentration of heavy particles in homogeneous isotropic turbulence. J Fluid Mech 256:27-68

Xiao J, Taylor PA (2002) On equilibrium profiles of suspended particles. Boundary-Layer Meteorol 105:471482 